\title{
Micro-Analysis System for Glucose with an Electrochemically Actuated Sampling Mechanism
}

\author{
Osamu Kawatoko* Non-member Rei Yoneyama* Non-member \\ Hiroaki Suzuki* Member
}

A micro-analysis system that imitates a mosquito was fabricated by incorporating an electrochemical micro-sampling mechanism that uses an electrochemically generated or shrunk hydrogen bubble. The rates of growth and shrinkage of the bubble could be controlled by applying a potential. An external standard solution could be successfully withdrawn through a syringe needle using the sampling mechanism. A distinct current increase was observed in the on-chip glucose sensor following the withdrawal of the standard solution. The calibration curve was linear for glucose concentrations up to $10 \mathrm{mM}$.

Keywords : Sampling mechanism, Bubble, Mosquito, Glucose

\section{Introduction}

In order to minimize the pain that accompanies blood sampling, analysis systems that can conduct less invasive analyses than the current procedures are desirable. In particular, this applies to the measurement of the blood glucose level of patients suffering from serious diabetes. Some micro-systems aiming for this style of sensing have been developed ${ }^{(1)(2)}$. In order to construct such a system, the essential components are a sensor, a sampling mechanism, and a microscopic needle. In this study, the system was constructed with a newly developed electrochemical sampling mechanism $^{(3)}$. It features low operating voltage and power consumption in addition to simple structure and function. The performance of the components and the entire system is reported.

\section{Experimental}

Three-electrode systems for sensing and actuation were formed on a glass substrate. Platinum was used for the working and auxiliary electrodes, and $\mathrm{Ag} / \mathrm{AgCl}$ was used for the reference electrodes. Their active areas were delineated with a polyimide layer. For the working electrode of the sampling mechanism, 6 pinholes of $0.1 \mathrm{~mm}$ in diameter were formed as active areas to effectively grow or shrink the bubble. Platinum black was grown only on the active areas. The electrodes for the sensor were formed near the end of a flow channel. The active area of the working electrode was $0.3 \mathrm{~mm} \times 0.3 \mathrm{~mm}$. Glucose oxidase (from Aspergillus Niger) was immobilized on the platinum working electrode with bovine serum albumin and glutaraldehyde (activity: 15.8 units $/ \mathrm{mm}^{2}$ ).

The flow channel and containers for the electrolyte solution were formed on a PMMA substrate. Structures formed on a thick-film photoresist SU-8 template were embossed into the PMMA substrate at $190^{\circ} \mathrm{C}$. For the sampling mechanism, the compartments for the three electrodes were separated and connected with liquid junctions (Fig. 1). The junctions and their periphery were filled with a hydrophilic resin (ENT-2000, Kansai Paint, Osaka) to suppress the influx of the hydrogen bubble. To minimize the Ohmic drop caused by the evolution of hydrogen gas, the bottom area of the cavity for the working electrode was partially filled with the same resin. The flow channel was formed

\footnotetext{
* Institute of Materials Science, University of Tsukuba
} 1-1-1, Tennodai, Tsukuba, Ibaraki 305-8573 from the compartment for the sampling mechanism to the exit. The glass and PMMA substrates were bonded with a photocurable adhesive (BENEFIX PC, Adell, Tokyo).

At the end of the flow channel, a needle was attached with an adhesive. Although the batch fabrication of needles of appropriate geometry is our current concern, a commercially available syringe needle of $0.4 \mathrm{~mm}$ in outer diameter was used here. The distance between the needle and the edge was $1.1 \mathrm{~mm}$. In other words, the dead volume was approximately $20 \mathrm{nl}$.

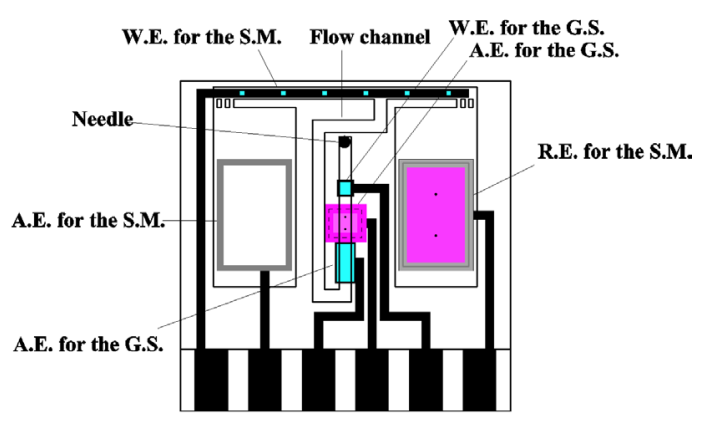

(a)

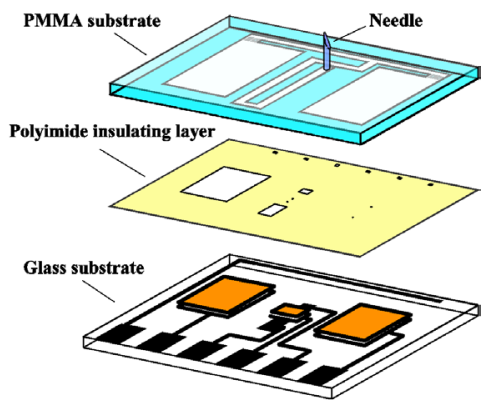

(b)

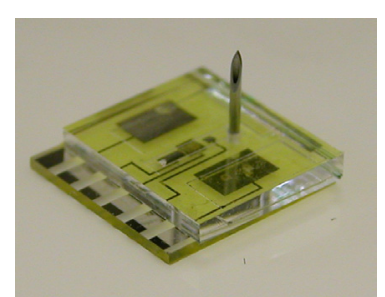

(c)
S.M.: sampling mechanism, G.S.: glucose sensor, W.E.: working electrode, R.E.: reference electrode, A.E.: auxiliary electrode. The dimensions of the chip are $8 \mathrm{~mm} \times 8 \mathrm{~mm}$.

Fig. 1. Structure of the system. (a) : plan view of the system showing the mutual relation between the electrodes and the flow channel, (b) : decomposed structure, (c) : completed system. 
A $0.1 \mathrm{M}$ phosphate buffer solution containing $0.1 \mathrm{M} \mathrm{KCl}(\mathrm{pH}$ 7.4) was used for the electrolyte solution. Although an acidic electrolyte solution was preferable to actuate the sampling mechanism, a neutral solution was used to avoid the denaturation of the enzyme and to minimize the $\mathrm{pH}$ change that accompanies the withdrawal of human blood.

Before using the system, the internal flow channels and the containers were filled with the electrolyte solution in a vacuum. The sampling mechanism was operated by growing or shrinking a hydrogen bubble on its working electrode. The rates of growth and shrinkage could be controlled by the working electrode potential. The potential was set at $-1.4 \mathrm{~V}$ (vs. on-chip $\mathrm{Ag} / \mathrm{AgCl}$ ) when flushing and at $+0.1 \mathrm{~V}$ when sampling. The potential of the working electrode for the sensor was set at $+0.7 \mathrm{~V}$. All experiments were conducted on a hotplate maintained at $37 \pm$ $0.1^{\circ} \mathrm{C}$.

\section{Results and Discussion}

Peculiar peaks originating from the adsorption and desorption of protons and molecular hydrogen were observed in cyclic voltammograms obtained using the platinum black working electrode for the sampling mechanism. The hydrogen bubble could be successfully grown or shrunk in the system. The movement of a dye solution withdrawn from the inlet was actually observed accompanying the change. Even if a bubble remained

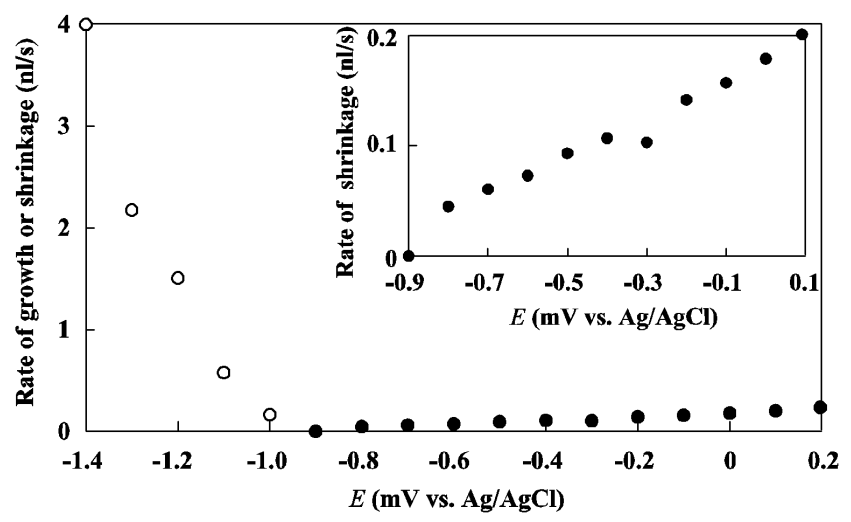

$\bigcirc$ : growth, $\bullet$ : shrinkage. The inset shows the expanded view of the right half. Fig. 2. Dependence of the rate of the growth and shrinkage of the bubble in the system.

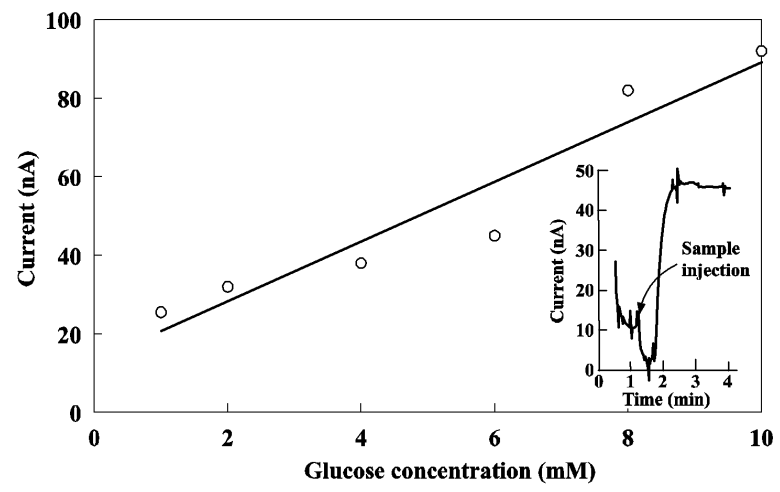

Fig. 3. Calibration plot for the glucose sensor in the system. The inset shows the variation of the current of the sensor after withdrawing a standard solution. near the inlet, pressure was effectively exerted through the solution column in the flow channel, and sampling could be conducted without any problems. The dependence of the rates of withdrawal and flushing (as the rates of the bubble volume change) is shown in Fig. 2. The rates were influenced by the $\mathrm{pH}$ of the electrolyte solution and the structure around the working electrode. The low sampling rate (shrinking ratio of the bubble) suggests that the electrolyte solution did not wet the working electrode well, an issue that needs more consideration.

In order to check the function of the system, the solution in the flow channel was first flushed. Then, a glucose standard solution was introduced, and the output current of the sensor was recorded. A distinct current increase was observed at $60 \sim 120 \mathrm{~s}$ after withdrawing the solution. Figure 3 shows the calibration curve obtained in the system by repeating the above-mentioned procedure. A linear relationship was confirmed up to $10 \mathrm{mM}$.

In handling real samples such as blood, the viscosity of the fluid is a point of consideration. The withdrawal of whole blood samples through a microscopic needle has successfully been done. Therefore, as long as the neelde is not clogged and the withdrawing pressure is effectively exerted by the shrinking bubble, sampling is expected to be as successful as it has been with the standard solutions. Measurements using real samples will be conducted in a future study.

\section{Conclusions}

Because of its simple structure and principle of operation, an electrochemical sampling mechanism is advantageous in a micro-system. Along with the on-chip glucose sensor, the function of the completed system was confirmed. In addition to the fabrication of a microscopic needle, other issues also remain to be analyzed, such as the immobilizaion of the enzyme and the formation of perm-selective and diffusion-limiting membranes on a very small area. We are currently developing the method, and the results will be reported elsewhere.

\section{Acknowledgment}

This study was supported by a Grant-in-Aid for Scientific Research on Priority Areas, by the 21st Century COE Program both under the Ministry of Education, Culture, Sports, Science and Technology, and by the Research for the Future Program of the Japan Society for the Promotion of Science.

(Manuscript received April 23, 2003, revised July 3, 2003)

\section{References}

(1) H. Suzuki, T. Tokuda, and K. Kobayashi : "A disposable "intelligent mosquito" with a reversible sampling mechanism using the volume-phase transition of a gel", Sensors and Actuators B, 83, p.53 (2002)

(2) K. Oka, S. Aoyagi, Y. Arai, Y. Isono, G. Hashiguchi, and H. Fujita : "Fabriaction of a micro needle for a trace blood test", Sensors and Actuators A, 97-98, p.478 (2002)

(3) H. Suzuki and R. Yoneyama : "A reversible electrochemical nano syringe pump and some considerations to realize low-power consumption", Sensors and Actuators B, 83, p.242 (2002) 